\title{
Bursting Dynamics of Thin Free Liquid Films from Newtonian and Viscoelastic Solutions
}

\author{
L. J. Evers, S. Yu. Shulepov,* and G. Frens \\ Laboratory of Physical Chemistry, Delft University of Technology, Julianalaan 136, 2628 BL Delft, The Netherlands
}

(Received 12 May 1997)

\begin{abstract}
The rupture velocity of a liquid film is usually obtained from an energy balance: Energy of the disappearing surface is transformed into kinetic energy of a moving rim, and dissipated due to the inelastic impact of this rim with a resting part of the film. This gives $\nu=(2 \gamma / \rho h)^{1 / 2}$ where $h$ is the film thickness, $\rho$ is the liquid density, and $\gamma$ is the surface tension. While thick films obey this behavior, thinner films can deviate remarkably: The velocity is drastically decelerated. This follows from the model developed, which accounts for elasticity when the thickness of a film becomes commensurate with (or smaller than) the mesh size, reflecting an enhanced structure. [S0031-9007(97)04779-0]
\end{abstract}

PACS numbers: $68.15 .+\mathrm{e}$

During the last decade, attention to confined liquid layers increased rapidly. This is explained by very different properties of these systems compared to their bulk analogies - thin and ultrathin films demonstrate an enhanced viscoelastic behavior (see, for example, [1-6]). In the present Letter, we investigate the bursting behavior of very thin films from Newtonian and viscoelastic solutions, which cannot be explained within the frame of existing theories.

In [4], we have improved the experimental setup of McEntee and Mysels [7], and reported a few new features of the rupture dynamics of very thin films from Newtonian and viscoelastic solutions. In those experiments, rupture was initiated by an electric spark, which triggered simultaneously a high-speed cine-photoflash. In the latter, a nanosecond pulse generator activates periodically a light flash with repeating capability of about $10^{4} \mathrm{~s}^{-1}$ with an accuracy within $2 \%$. With our setup, one can follow the evolution of a hole in a liquid film on one single photo. This allows observing additional fine details of the dynamic rupture process, which were not reported before. We have carried out experiments with free liquid films drawn from Newtonian, sodium dodecylsulfate (SDS) solutions and from viscoelastic cetyl-trimethyl ammonium bromide (CTAB-gel) solutions as well. Films from CTAB-gel up to $\sim 1 \mu \mathrm{m}$ thickness burst with a significantly lower velocity than that predicted by Culick [8], but for thicker films a transition to a Culick-like behavior occurs. The main new feature, that all CTAB-gel films demonstrate, is a deceleration of the rupture process. This deceleration becomes more pronounced as the film thickness decreases. Also, films of the "equilibrium" thicknesses have a scalloped appearance of the rim, which was discussed in more detail in [4,5].

In Fig. 1, a typical result of experiments with the Newton-black film of a Newtonian solution (a) and of a CTAB-gel solution (b) as well, is presented. The pictures closely resemble each other, suggesting that both systems on this length scale have many common features, which determine the overall macroscopic behavior of these very thin films. From such a photograph, the hole radius as a function of time can readily be extracted (see Fig. 2).

Usually, writing an energy (impulse) balance for such a system, it is assumed that the energy of a "disappearing" surface (which is expressed in terms of the surface tension) transforms partially into the kinetic energy of a moving rim. Also, a part of the energy dissipates during impact of this rim with a still-standing part of the film [8,9]. However, the treatment of gel-like systems implies an incorporation of the elasticity into a balance equation describing deformation of a confined molecular network. As it will be seen later, the experimentally analogous behavior of both systems creates a basis for the unified description, where the mesh size in confined physical gel phase can directly be compared with the characteristic length describing correlations arising in molecular bilayers (the Newton-black films, for instance).

We write after Pandit and Davidson [9] an energy balance equation, where, however, the air drag effect (introduced in [10]) and elastic deformation are also taken into account:

$$
\begin{gathered}
d E_{\mathrm{surf}}=d E_{\mathrm{kin}}+d E_{\mathrm{diss}}+d E_{\mathrm{drag}}, \\
d E_{\mathrm{surf}}=d\left(2 \gamma \Delta \vartheta \frac{1}{2}\right), \quad d E_{\mathrm{kin}}=d\left(\frac{m \nu^{2}}{2}\right), \\
d E_{\mathrm{diss}}=\nu^{2} / 2 d m, \quad m=\rho \Delta V,
\end{gathered}
$$

where $m$ is the mass of a moving film element, $\Delta V$ is the volume of this film element, $\rho$ is the density of a liquid, and

$$
\begin{gathered}
d E_{\text {elast }}=d\left(\frac{1}{2} \Delta V G\left[\lambda_{1}^{2}+\lambda_{2}^{2}+\lambda_{3}^{2}-3\right]\right), \\
d E_{\text {drag }}=F_{\text {drag }} d R=\frac{1}{2} C_{D} \nu^{2} \rho_{\text {atm }} \sqrt{\frac{R^{3} h}{a}} \Delta \vartheta d R,
\end{gathered}
$$

where $\Delta V=h R^{2} \Delta \vartheta \frac{1}{2} . \quad \lambda_{i}$ is an extension ratio in the $i$ th direction. A radial coordinate system with the origin at the center of a hole is used. We must notice here that a simple, neo-Hookian response of the material is assumed. Further, we model the deformation of a film element to the shape of the moving rim by a simplified geometry as 


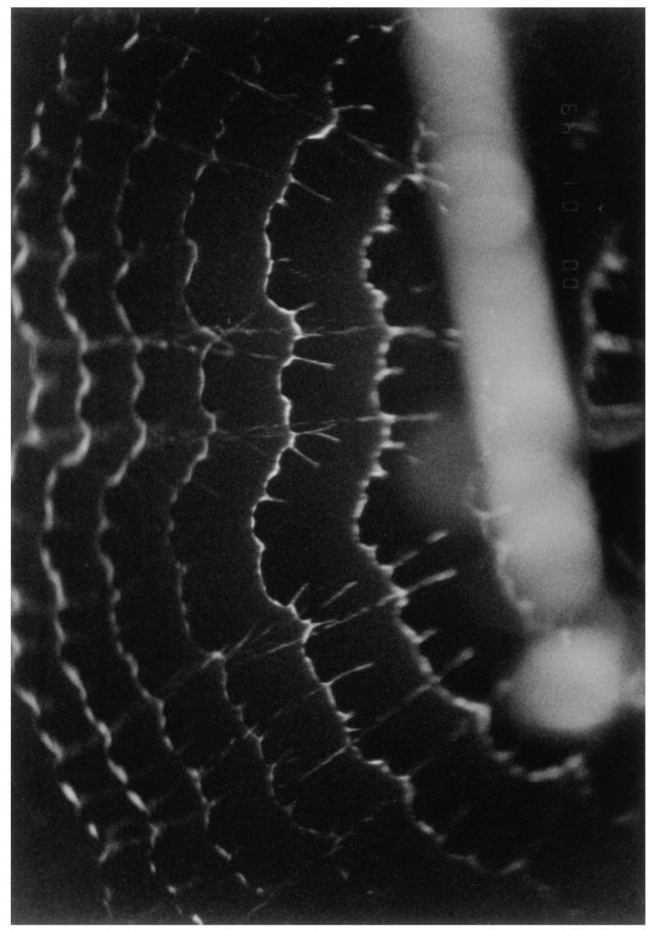

(a)

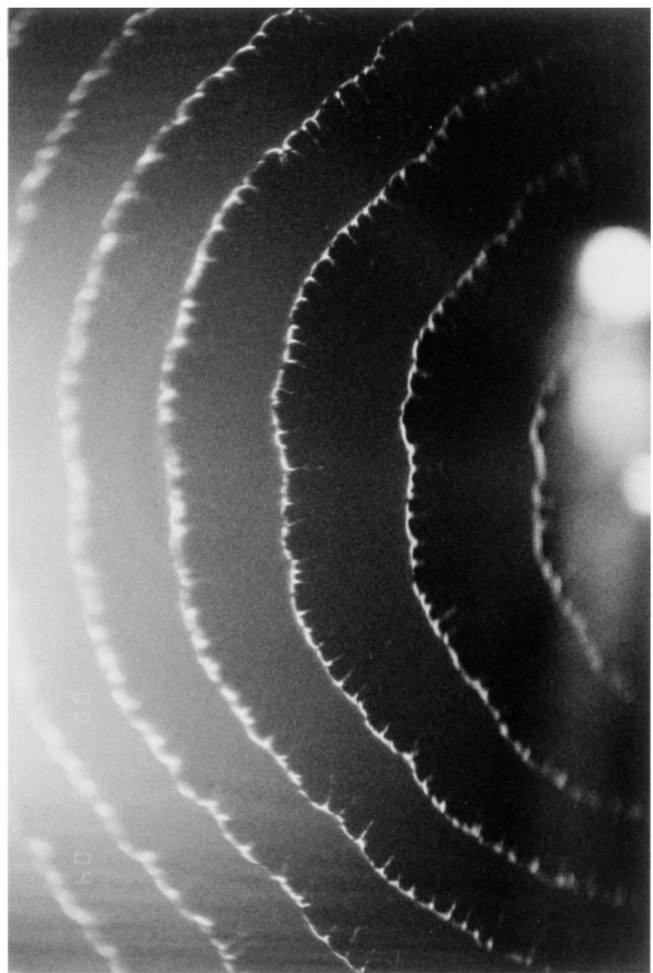

(b)

FIG. 1. Bursting Newton-black films drawn from SDS (a) and CTAB-gel (b) solutions. Photos show a "stroboscopic movie" of a hole expanding in a liquid sheet with thickness $h \approx 5 \mathrm{~nm}$.

is illustrated by Fig. 3. This corresponds to a pure shear deformation. Since for very thin films condition $h \ll R$ for $R$ 's of an interest is always fulfilled $\left(\lambda_{3} \gg 1\right)$, Eq. (1) is reduced to

$$
\begin{aligned}
4 \gamma R= & 2 \nu^{2}(R) R \rho h+\frac{1}{2} R^{2} \rho h \frac{d}{d R}\left[\nu^{2}(R)\right]+\frac{d}{d R} \\
& \times\left[\frac{1}{2} R^{2} h G \lambda_{3}^{2}\right]+\frac{1}{2} C_{D} \nu^{2}(R) \rho_{\mathrm{atm}} \sqrt{\frac{R^{3} h}{a}} .
\end{aligned}
$$

Actually, $C_{D}$ is a function of the Reynolds number $(\operatorname{Re}=\nu d / \mu$, where $d(R)$ is the rim diameter and $\mu$ is the kinematic viscosity). An approximate analytical expression for the geometry we are dealing with is known for rather low and large Reynolds numbers only. However, the interval $1<\operatorname{Re}<100$ is typically a field of numerical calculations [11]. Under conditions of our experiment, a characteristic Reynolds number is falling into this intermediate region ( $\operatorname{Re} \sim 2-5$ ), and this makes Eq. (2) quite complex. Fortunately, the rupture velocity decays with $R$, whereas the characteristic size of the rim grows. These two effects compensate each other, and $C_{D}$ remains nearly constant. One can see that only terms of the highest order with respect to $R / h$ make a significant contribution into the elastic component [Eq. (2)], and, as a consequence, the choice of a proper rheological model is not really so important.

In Eq. (2) $G$ is the relaxation modulus of a film, that is for neo-Hookian response given by

$$
G=G_{r}+\int_{0}^{\infty} d \tau H(\tau) \exp (-t / \tau)
$$

where $G_{r}$ is the equilibrium modulus, and $H(\tau)$ is the relaxation spectrum. If we assume, for the sake of brevity, that the relaxation spectrum consists of one relaxation time $(\tau)$ only [for a wormlike micellar phase (CTAB-gel) this is generally accepted [12]], Eq. (3) can be reduced to

$$
G \cong G_{0}[g+\exp (-t / \tau)],
$$

where $G_{0}$ is the plateau modulus, and, usually, $g=$ $G_{r} / G_{0} \ll 1$. Finally, we write

$$
\begin{aligned}
\frac{d w}{d R}+\frac{4}{R} w(1+ & \left.C_{D} \frac{\rho_{\mathrm{atm}}}{\rho} \sqrt{\frac{R}{a h}}\right)- \\
\frac{4}{R}\left(\frac{2}{\rho h}-\frac{3}{2 a} \frac{G R}{\rho h}\right) & =0,
\end{aligned}
$$

where $t=\int d R / \nu(R)$, and $w=\nu^{2}(R)$.

At the very beginning of the rupture process $(t \ll \tau)$, Eq. (4) can be solved analytically if the drag effect is dropped, yielding

$$
\nu=\sqrt{\frac{2 \gamma}{\rho h}-\frac{6}{5 a} G_{0}(h) \frac{R}{\rho h}} .
$$

This clarifies a role of the elastic term in the dynamic process.

To describe the explicit dependence of $\nu$ on the hole radius $R$ and the film thickness $h$, we must know dependence of the elasticity modulus $G$ on the properties 


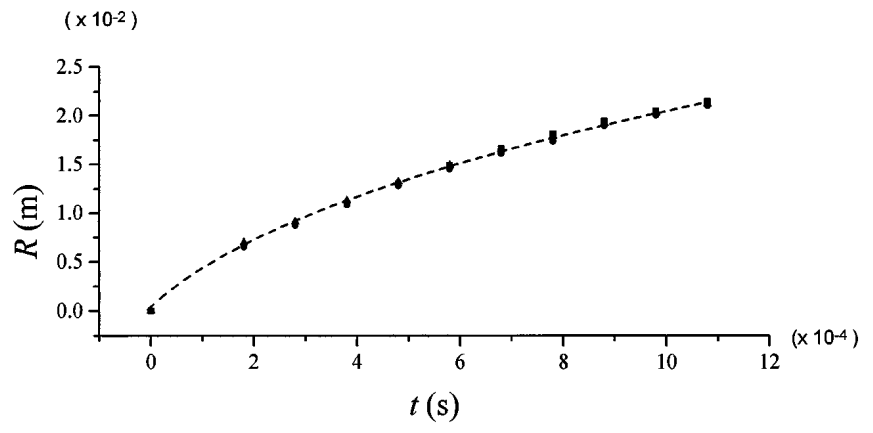

FIG. 2. The hole radius versus the time after nucleation of this hole. Typical results are shown for Newton-black films from an SDS solution. The dashed line indicates a trend of the dynamic process.

of a film. Following descriptions based upon a scaling concept, the bulk value of this characteristic is defined by so-called mesh size $\xi$ in a networklike system $[13,14]$ as

$$
G_{0} \sim k_{B} T / \xi^{d},
$$

where $d$ is the topological dimension of this system (in our case, $d=3$ ).

However, if such a system is confined into a restricted volume, the relation between this characteristic mesh length $\xi$ in the bulk phase and the characteristic size of that volume (in our case, $h$ ) is crucial [15]. When $h \gg \xi$, the bulk properties preserve; however, if $h$ becomes much smaller than $\xi$, the film thickness rather than the bulk mesh size determines elastic properties of the confined network. In the latter situation, when modeling the system behavior, the structure of a network at the interface must be known. Thus, dependence of the elasticity modulus on the film thickness $h$ may generally be described by a scaling relation

$$
G_{0} \sim 1 / h^{x},
$$

where $x$ is an exponent, which, actually, reflects the structure of a molecular network close to the interface. The systems under investigation are attributed to two limit-

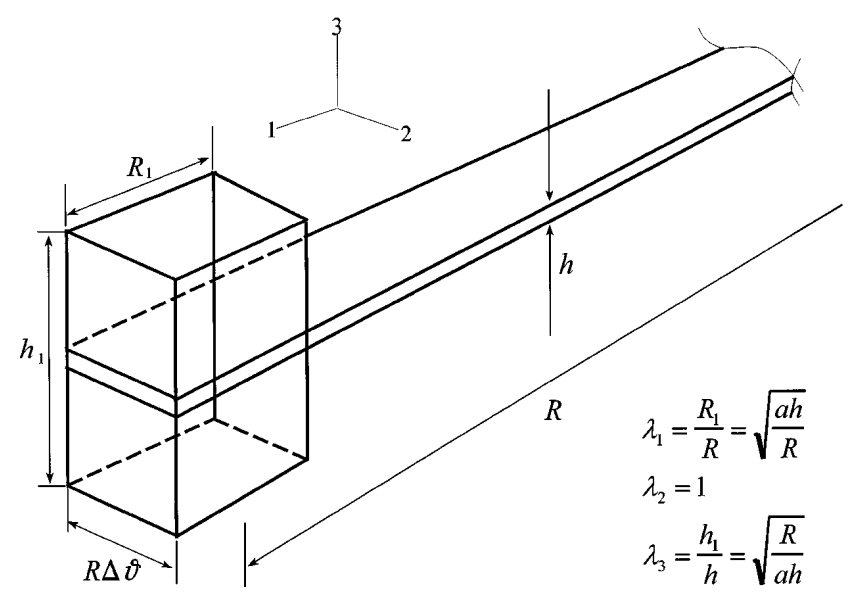

FIG. 3. A sketch of the model geometry of the deforming rim. ing cases. CTAB-gel is assumed (in zero approximation) to retain its bulk mesh size in the film plane. But in the perpendicular (to the film plane) direction, the characteristic size determining structural properties is commensurate with the film thickness ("chains" weakly attached to the surface). The Newton-black films drawn from an SDS solution are supposed to resemble the bilayer of a selfsimilar grid. In this way, dependence of the elastic modulus in these 2D-similar systems becomes $G_{1} \sim 1 / \xi^{2} h$ and $G_{2} \sim 1 / h^{3}$, respectively. The proportionality constant in the first case can be obtained from data on CTAB-gels in the bulk phase (see [16]); i.e., when $h$ becomes commensurate with $\xi, G_{1}$ has to acquire its bulk value. The situation with SDS bilayers is more complicated. There is an experimental evidence of enhanced elastic properties of Newtonian fluids confined on a nanometer scale [1,2]; however, a procedure to extract the needed constants from experiments is not established well, because such systems do not reveal the bulk elasticity. In this way, $G_{2}$ can be obtained by matching the numerical solution of our model with experimentally acquired data. This constant has been found from one experimental point, and then the whole velocity map (as a function of $R$ and $h$ ), is reconstructed.

Equation (4) can be recast to a form more suitable for numerical calculations. Having substituted $\nu=d R / d t$, we wrote the equation for $R$ as a function of $t$ :

$$
\begin{aligned}
\frac{d^{2} R}{d t^{2}}+\frac{2}{R}\left(\frac{d R}{d t}\right)^{2}\left(1+C_{d} \frac{\rho_{\mathrm{atm}}}{\rho} \sqrt{\frac{R}{a h}}\right)-\frac{2}{R} \times \\
\left(\frac{2 \gamma}{\rho h}-\frac{3}{2 a} \frac{R}{h} \frac{G_{0}^{*}}{\xi^{3}(h)} \exp (-t / \tau)\right)=0 .
\end{aligned}
$$

This equation has to be accompanied by appropriate boundary conditions. In our experiments, a hole of a finite size (typically, of about a micron) is created. The driving force-the surface tension-gives as an initial velocity of the hole propagation the Culick velocity; i.e., we may take $R(0)=\varepsilon, \nu(\varepsilon)=\nu_{\text {Culick }}$, where $\varepsilon \sim 1 \mu \mathrm{m}$.

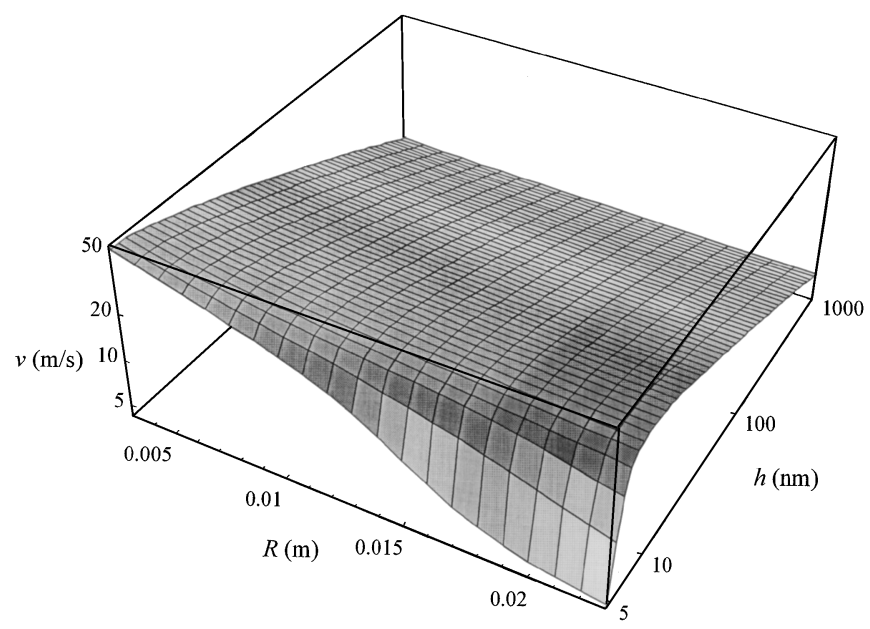

FIG. 4. The velocity of an expanding hole as a function of the film thickness $h$ and the hole radius $R$. 


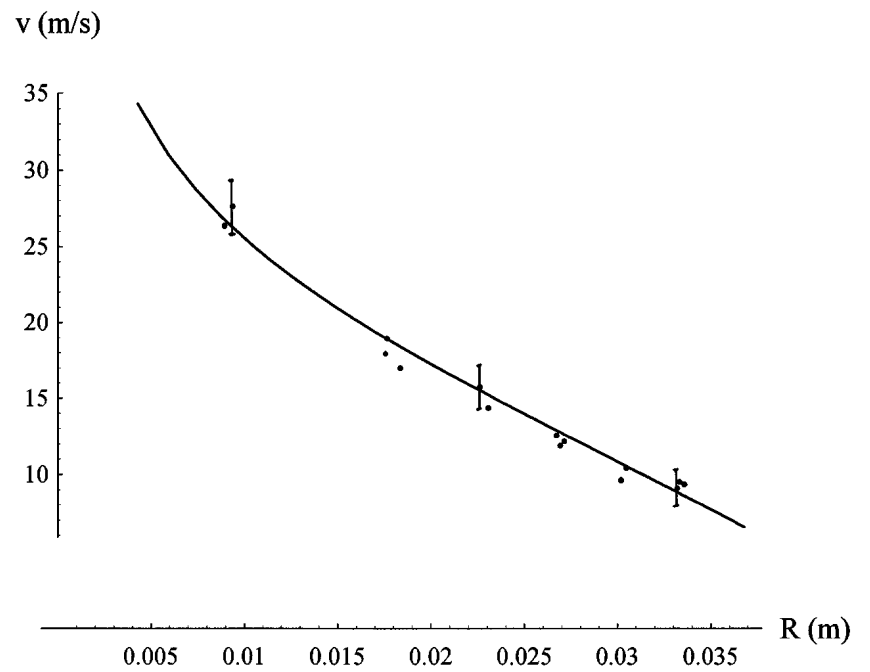

FIG. 5. The rupture velocity as a function of the hole radius. Points represent the experimental results for Newton-black films of a CTAB solution. The line is the solution of Eq. (8), where $\tau=0.015 \mathrm{sec}, C_{D}=7$, and $G_{1}=1.1 \mathrm{~N} / \mathrm{m}^{2}$ at $T=25^{\circ} \mathrm{C}$. $\xi$ in the bulk phase was taken $100 \mathrm{~nm}$.

On the basis of numerical calculation, behavior of the rupture velocity as a function of $R$ and $h$ can be obtained. This is illustrated by a three-dimensional plot drawn in Fig. 4 for an SDS system. The intercept of this plot with the $y-z$ plane on the background gives the dependence of the rupture velocity on the film thickness at the very beginning of the process, i.e., for very small $R$ 's. This behavior resembles a Culick-like dependence corrected for the air drag effect [10]. The intercept of this surface with the $y-z$ plane on the foreground illustrates the dependence of the rupture velocity on the film thickness, which was reported in the literature previously $[7,10]$,

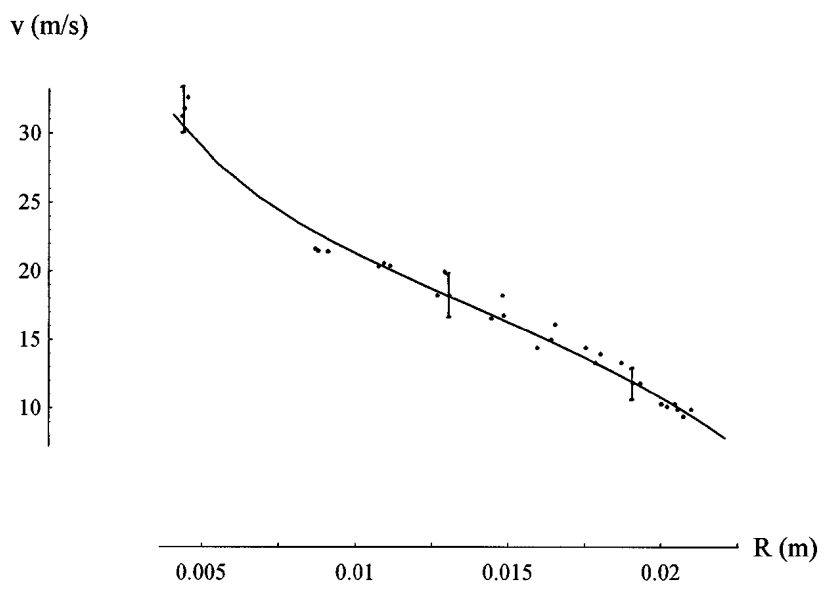

FIG. 6. The rupture velocity as a function of the hole radius. Points are the experimental results for Newton-black films from an SDS solution. The line is the solution of Eq. (8), where $\tau=0.03 \mathrm{sec}, C_{D}=7$, and $G_{2}=1.7 \mathrm{~N} / \mathrm{m}^{2}$ at $T=25^{\circ} \mathrm{C}$. and is typical for a rather large radius of the hole. The intercept with the $x-z$ plane on the side of thick films demonstrates a very slight sensitivity of the rupture velocity to the hole radius, i.e., typically Culick's behavior with the air drag correction. For the Newton-black film, the rupture velocity depends significantly on the hole size. This is a new result, which deserves more attention. We compare computations carried out on the basis of our theoretical modeling with the experimental data in Figs. 5 and 6 , where the rupture velocity of a Newton-black film is plotted against the radius of the propagating hole for CTAB-gel and SDS solutions, respectively. It is obvious that the elastic term which is proportional to the volume of the growing rim is responsible for such a deceleration.

A rather primitive model has been used when describing the elastic response of a confined molecular network. As one can see, this model reproduces quite well the qualitative behavior of the rupture velocity as a function of $R$ and $h$, and it has a rather good overall quantitative agreement with experimental observations. However, because of a very primitive geometry used in our modeling, and the relaxation spectrum being cut down, some fine details of the rupture process cannot be rendered, revealing the shortcomings of our approach.

*Corresponding author. Current address: Department of Solid State Chemistry and Materials Sciences, Eindhoven University of Technology, Postbus 513, 5600 MB Eindhoven, The Netherlands.

[1] J. Van Alsten and S. Granick, Phys. Rev. Lett. 61, 2570 (1988).

[2] A. Dhinojwala and S. Granick, J. Chem Soc. Faraday Trans. 92, 619 (1996).

[3] G. Debrégeas, P. Martin, and F. Brochard-Wyart, Phys. Rev. Lett. 75, 3886 (1995).

[4] L.J. Evers, S. Yu. Shulepov, and G. Frens, Faraday Discuss. 104, 335 (1996); 104, 360 (1996).

[5] L. J. Evers, E. J. Nijman, and G. Frens (to be published).

[6] P. G. de Gennes, Faraday Discuss. 104, 1 (1997).

[7] W. R. McEntee and K. J. Mysels, J. Phys. Chem. 73, 3018 (1969).

[8] F. E. C. Culick, J. Appl. Phys. 31, 1128 (1960).

[9] A. B. Pandit and J.F. Davidson, J. Fluid Mech. 212, 11 (1990).

[10] G. Frens, J. Phys. Chem. 78, 1949 (1974).

[11] G. K. Batchelor, An Introduction to Fluid Dynamics (Cambridge University Press, Cambridge, 1994).

[12] J.-F. Berret, Langmuir 13, 2227 (1997).

[13] M. Daoud and A. Coniglio, J. Phys. A 14, L301 (1981).

[14] J.E. Martin and D. Adolf, Annu. Rev. Phys. Chem. 42, 311 (1991).

[15] P. G. de Gennes, Scaling Concepts in Polymer Physics (Cornell University Press, Ithaca, 1993).

[16] T. A. Strivens, Colloid Polym. Sci. 267, 269 (1989). 